\title{
AN EMERGING TREND OF FEATURE EXTRACTION METHOD IN VIDEO PROCESSING
}

\author{
R.Revathi ${ }^{1}$ and Dr.M.Hemalatha ${ }^{2}$ \\ 1 \\ Research Scholar, Karpagam University, Coimbatore, TamilNadu,India. \\ s.revathisenathaegmail.com \\ ${ }^{2}$ Head Dept of Software Systems, Karpagam University, Coimbatore, \\ TamilNadu, India. \\ hema.bioinf@gmail.com
}

\begin{abstract}
Recently the progress in technology and flourishing applications open up new forecast and defy for the image and video processing community. Compared to still images, video sequences afford more information about how objects and scenarios change over time. Quality of video is very significant before applying it to any kind of processing techniques. This paper deals with two major problems in video processing they are noise reduction and object segmentation on video frames. The segmentation of objects is performed using foreground segmentation based and fuzzy c-means clustering segmentation is compared with the proposed method Improvised fuzzy $c$ - means segmentation based on color. This was applied in the video frame to segment various objects in the current frame. The proposed technique is a powerful method for image segmentation and it works for both single and multiple feature data with spatial information. The experimental result was conducted using various noises and filtering methods to show which is best suited among others and the proposed segmentation approach generates good quality segmented frames.
\end{abstract}

\section{Keywords}

Image Processing, Video Processing, Denoising and Filters, color segmentation, foreground color, background color and Feature Extraction.

\section{INTRODUCTION}

\subsection{Video Processing}

Video processing is used for the sound recorded in the video files and editing the image accordingly. By using various filter algorithms the user can perform editing functions. The output can be performed in frame by frame or in larger batches.

With the help of universal standard bus (USB) cable or fire wire attachment the recording device is used to extract the video files. The softwares and peripheral devices are used to load these files into the computer. In many PC modems are created with limited level of software which allows the user to compile images and video, etc.By using the combination of pre filters, intra filters, and post filters signal processing is also involved [1].

David C. Wyld, et al. (Eds): CCSEA, SEA, CLOUD, DKMP, CS \& IT 05, pp. 69-80, 2012. (C) CS \& IT-CSCP 2012 


\subsection{Video Denoising}

The method for removing the noise from video signal is termed as video denoising.These methods are divided into spatial, temporal and spatio-temporal.In this paper spatial video denoising methods are used. Noise reduction is used in each frames of video. Due to noise the images are disturbed and noise removal in video processing takes place. The result of noise removal takes the quality of the video processing technique. Various techniques for removing the noise are identified in color image processing.

Depending upon the types of image noise interpreted the noise removal problem occurs. Mainly Linear and Non-linear filtering is used for this type of noise reduction. Compared to non linear filter linear filter is not much effective since the tendencies of the blur image at the edges are removed effectively. But in non linear filter is solves this problem. For the past years non linear filters are used in classical and fuzzy techniques. Here the classical filter removes the blur edges concurrently and fuzzy filter combine and produce the edge preservation and smoothening. This paper provides the results for different filtering techniques and these are compared. The goals of this paper are to find a preprocessing method based on noise removal.

\section{IMAGE DENOISING}

A set of data exists in many ways of denoise. The properties of an image denoising model are that it removes preserving edges noise. Mostly Linear Models are used for Image denoising. Most common approach is that we use Gaussian filter, or consistently solving the equation of heat- equation with the noisy image as input-data.

Advantage: High speed is used.

Disadvantage: They are not able to save edges in a good manner: i.e. edges, which are identified are non continuities in the image, are smeared out.

\subsection{Types of Noise}

- Amplifier Noise (Gaussian Noise)

- Salt-and-Pepper Noise

- Periodic Noise

\subsection{Types of Filters}

- Average Filter

- Median Filter

- Wiener Filter

- Rank Order Filter

- Gaussian Filter

- Non-Linear Filter

- Outlier Filter 


\subsection{Testing With Noise}

To calculate the performance of noise in a video, we use three types of noises. By adding the various types of noise we clean the image source so as to get the extract parameters of added noise. By filters initialization we get the results. To videos various noises are applied and filtered. The results are compared with the original image. The original video shows $120 X 160$ grayscale image. The noisy image produced by various noise filters is shown in Fig(b).While applying salt and pepper noise, the filter which is shows the accuracy is shown in Fig (c)\& (d).

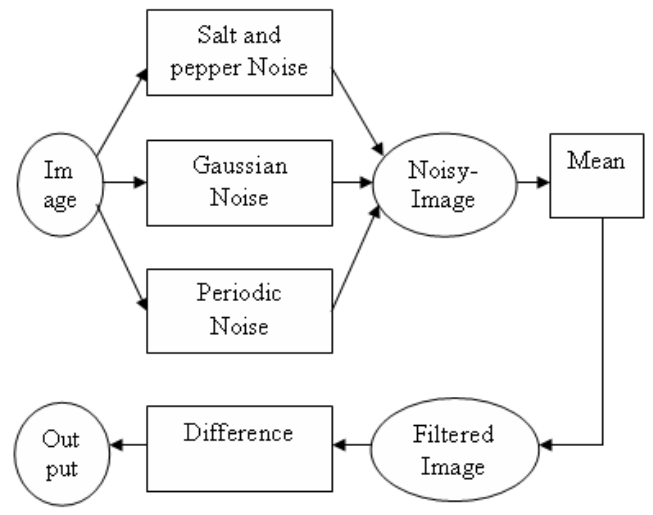

Figure 1: Evaluation of Filter Performance

To the video, when Gaussian noise is applied and filtered Wiener filter shows the accuracy difference of the image as shown is the Fig(e).To periodic noise filter clean the 2D median filter shows the accuracy and is chosen in Fig(f).The Difference Image Filter is applied to compare the filtered image and the original image. The MSE (Mean Square Error) differences were calculated for various noises and are obtained as follows. For salt and pepper noise it is 255.9669 and 187.3607, for Gaussian Noise the calculated value is 175.3028 and for periodic noise 150.0259.

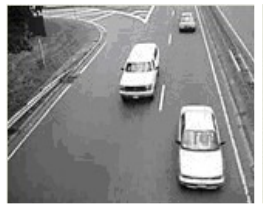

(a)

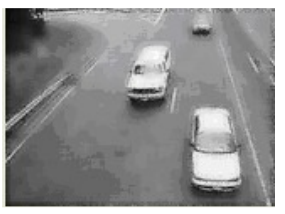

(b)

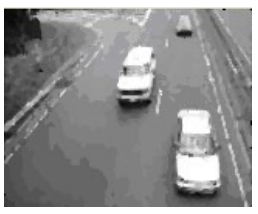

(c)

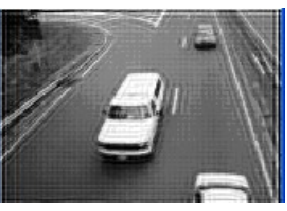

(d)

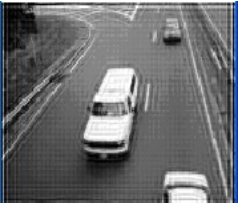

(e)

Figure 2: Filtering noise from an image. (a) Original image, (b) Noisy image, (c) Wiener filtered image, (d) Median filtered Image (e) 2D Filter Image

\section{SEGMENTATION}

Segmentation is the process of separating an image of digital into multiple æames in terms of sets of pixels, also known as super pixels. The aim of segmentation is to make things easier and/or modify the presentation of an image into some meaningful and easier to examine [12].

Segmentation of an image is used to locate the object of an image and its boundaries like lines, curves, etc. The method of passing a label to every pixel in an image that pixels with the same label share certain visual characteristics is done by Image 
Segmentation. Segmentation is used for object recognition, occlusion boundary evaluation within action or stereo systems, image compression, image editing, or image database look-up.

\subsection{Fuzzy C-Means}

The data of particular set have the member of different values and various clusters is termed as Fuzzy C-Means.

\subsection{Color Image Segmentation}

Segmentation similar object can be represented using less number of bits. In applications, such as storage of turner information based on coloring, segmentation is an effective method. Using color, segmentation becomes simple and faster compared to monochrome processing. Color thus adds a new dimension to visual processing. With the colors of interest in the image computer is trained in the form of mean and standard deviation. Estimation of parameters from samples can be done based on the methods of moments.

\subsection{Foreground Segmentation}

Object segmentation from a video sequence, one important problem in the image processing field, includes such applications as video surveillance, teleconferencing, video editing, humancomputer interface, etc. An initial foreground mask is constructed by background difference using multiple thresholds. The initial foreground region is composed of four categories based on their reliability. Then shadow regions are eliminated using color components, and each object is labeled with its own identification number. In the fourth step, to smoothen the boundaries of foreground and to eliminate holes inside the regions, we use a silhouette extraction technique on each object. However, the silhouette extraction technique covers all regions inside the object silhouette even including real holes in the object. Finally we recover real holes in the object using a region growing technique and generate final foreground masks.

\subsection{Background Subtraction}

Once images are taken, the system performs a background subtraction of the image to isolate the person and create a mask. The background subtraction involves in two steps. First, step involves the channel wise subtraction of the pixel from the background image to foreground image. The resulting channel differences are summed, and the threshold is calculated according to the image which contain white are masked else it is set to black.

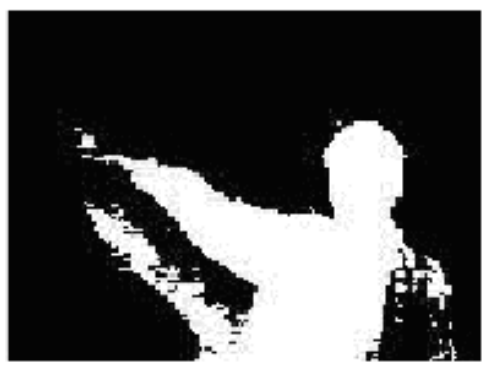

Figure 3: A background Subtraction mask

The results shown in the image is a mask that the outlines the body of the person (Figure 3).The two important featured of the image are the reality of a second right "arm", which is the result of a shadow of the right arm falling on the wall behind the person, and the noise in the generat 
ed mask image.This phantom arm is the result of the poor image quality of the input image, but could be corrected for by space of the conversion of the color images and the use of another method of background subtraction.

If the images were converted from RGB to HSB color space, then the subtracted values of the pixels(before being set to white or black, could be inspected, and those pixels that have a very low brightness could be discarded as well( set to black).since a shadow tends to be very dark when compared to the body of a person ( in an image),those pixels that have a low brightness can be inferred to be part of a shadow, and therefore unimportant(discard able).The noise in the mask image for the GRS has the function that compares a pixel tot eh surrounding pixels(of the given radius),and sets that pixel to the value(black or white) of the majority of the other pixels. The GRS runs two such filters over the mask data, one with the radius of one pixel and another of the radius of three pixels.

\subsection{Fuzzy Image Segmentation}

A branch of logic that uses degrees of membership in sets rather than a strict true/false membership.

- A tool to represent imprecise, ambiguous, and vague information

- Its power is the ability to perform meaningful and reasonable operations

- Fuzzy logic is not logic that is fuzzy -- it is logic of fuzziness.

- It extends conventional Boolean logic to recognize partial truths and uncertainties.

\section{FEATURE EXTRACTION}

The techniques used for extracting information from an image are known as Image Analysis. An image is composed of edges and shapes of grey. Edge is corresponding to fast change in grey level and corresponds to high frequency information. Shade is corresponding to low frequency information.

Separation of high frequency information means edge detection. An edge or boundary in the external information of image. The internal features in an image can be found using segmentation and texture. These features depend on the reflectivity property.

Segmentation of an image means separating certain features in the image. While treating other part as a background if the image consists of a number of features of interest then we can segment them one at a time.

Texture of an image is quantitatively described by its coarseness. The coarseness index is related to the spatial repetition period of the local structure. Image feature is a distinguishing characteristic of an image. Spectral and spatial domains are the main methods used for feature separation. Motions of an object are studied from study of multiple images, separated by varying periods of time.

Image Analysis is quite different from other image processing operations such as restoration, enhancement and coding, where output is another image. Image Analysis basically involves the study of features extraction, segmentation and classification techniques. 


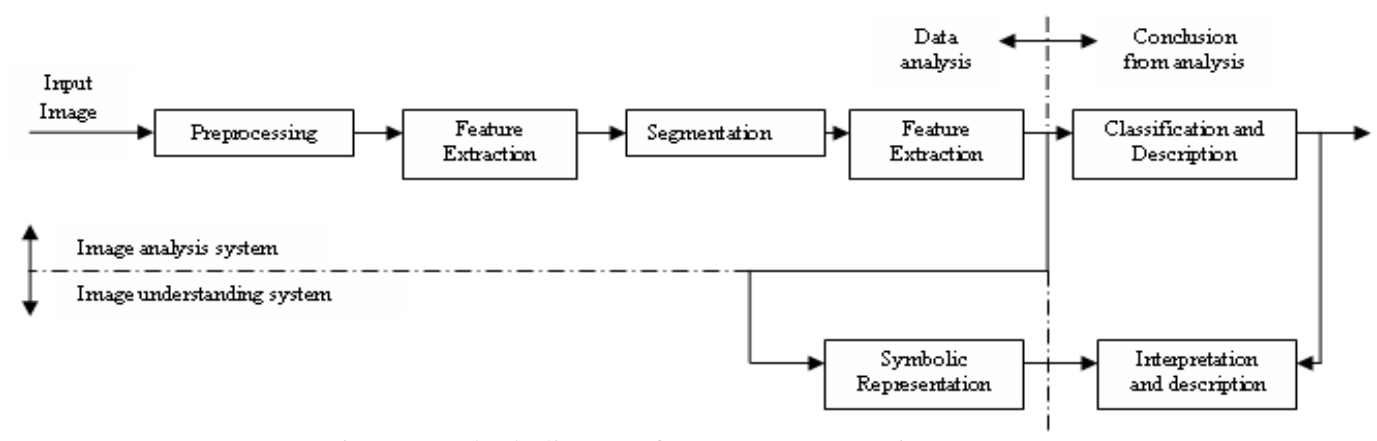

Figure 4: Block diagram for Feature Extraction Process

The input image is first preprocessed which may involve restoration, enhancement or just proper representation of the data. Certain features are extracted for segmentation of image into its components. The segmented image is fed into a classifier or an image understanding system. Image classification maps different regions or segments into one of several objects each identified by a label. Image understanding system determines the relationship between different object in a screen in order to provide its description.

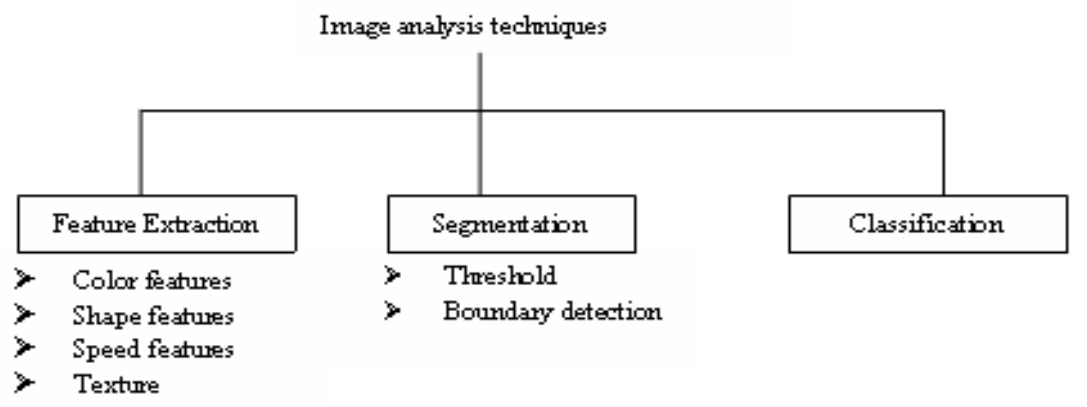

Figure 5: Analysis Techniques for Feature Extraction

\subsection{Edge based Segmentation:}

Edge can be defined as a set of connected pixels that lies in the boundary between two regions.

Shape descriptor is a set of numbers that are produced to represent a given shape feature. A descriptor attempts to quantify the shape in ways that agree with human intuition (or task-specific requirements). Good retrieval accuracy requires a shape descriptor to be able to effectively find perceptually similar shapes from a database. Usually, the descriptors are in the form of a vector. We classify the various features currently employed as follows:

- General features: Application independent features such as color, texture, and shape. According to the abstraction level, they can be further divided into:

- Pixel-level features: Features calculated at each pixel, e.g. color, location.

- Local features: Features calculated over the results of subdivision of the image band on image 


\subsection{Segmentation or edge detection.}

Global features: Features calculated over the entire image or just regular sub-area of an image.

- Domain-specific features: Application dependent features such as human faces, fingerprints, and conceptual features.

These features are often a synthesis of low-level features for a specific domain.

\subsubsection{Color:}

The color feature is one of the most widely used visual features in image retrieval. Images characterized by color features have many advantages:

- Robustness. The color histogram is invariant to rotation of the image on the view axis, and changes in small steps when rotated otherwise or scaled [14]. It is also insensitive to changes in image and histogram resolution and occlusion.

- Effectiveness. There is high percentage of relevance between the query image and the extracted matching images.

- Implementation simplicity. The construction of the color histogram is a straightforward process, including scanning the image, assigning color values to the resolution of the histogram, and building the histogram using color components as indices.

\subsubsection{Texture:}

Texture is another important property of images. Texture is a powerful regional descriptor that helps in the retrieval process. Texture, on its own does not have the capability of finding similar images, but it can be used to classify textured images from non-textured ones and then be combined with another visual attribute like color to make the retrieval more effective.

Texture has been one of the most important characteristic which has been used to classify and recognize objects and have been used in finding similarities between images in multimedia databases.

Basically, texture representation methods can be classified into two categories: structural; and statistical. Statistical methods, including Fourier power spectra, co-occurrence matrices, shiftinvariant principal component analysis (SPCA), Tamura features, World decomposition, Markov random field, fractal model, and multi-resolution filtering techniques such as Gabor and wavelet transform, characterize texture by the statistical distribution of the image intensity.

\subsubsection{Shape}

Shape based image retrieval is the measuring of similarity between shapes represented by their features. Shape is an important visual feature and it is one of the primitive features for image content description. Shape content description is difficult to define because measuring the similarity between shapes is difficult. Therefore, two steps are essential in shape based image retrieval, they are: feature extraction and similarity measurement between the extracted features. Shape descriptors can be divided into two main categories: region based and contour-based methods.

Region-based methods use the whole area of an object for shape description, while contour-based methods use only the information present in the contour of an object 


\section{PROPOSED WORK}

For color video frames with RGB representation, the color of a pixel is a combination of the three primal colors red, green, and blue. RGB is suitable for color display, but not good for color scene segmentation and analysis because of the high correlation among the R, G, and B components. By high correlation, we mean that if the intensity changes, all the three components will change accordingly. In this context, color image segmentation using evidence theory appears to be an attractive method. However, to fuse different images using fuzzy c means approach, the appropriate determination of member ship function plays a crucial role, since assignation of a pixel to a cluster is given directly by the estimated membership functions In the present study, the method of generating the membership functions is based on the assumption of a Gaussian distribution. To do this, histogram analysis is applied on the color feature domains. These are used to extract homogeneous regions in each primitive color. Once the mass functions are estimated, the fuzzy c means combination rule is applied to obtain the final segmentation results.

\section{Segmentation of Train:}
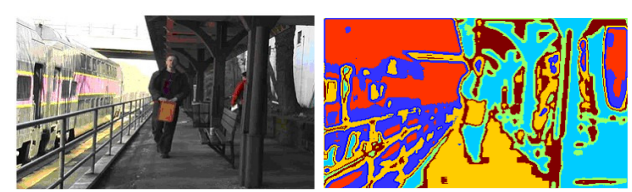

Input Image

Segmentation Maps
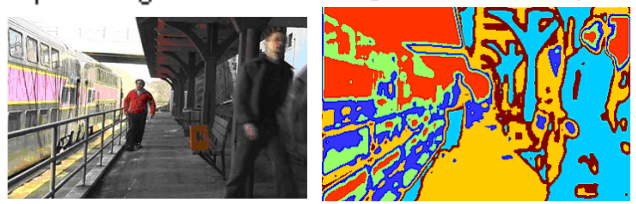

Segmentation of Traffic:
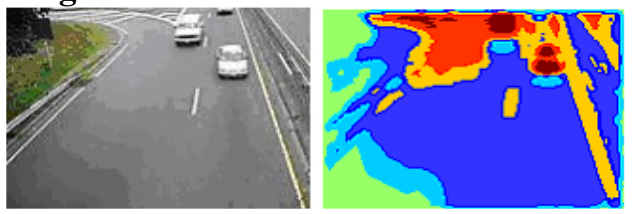

Input Image

Segmentation Maps
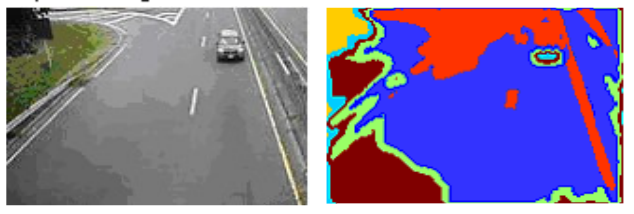

Input Image

Segmentation Maps

Foreground segmentation
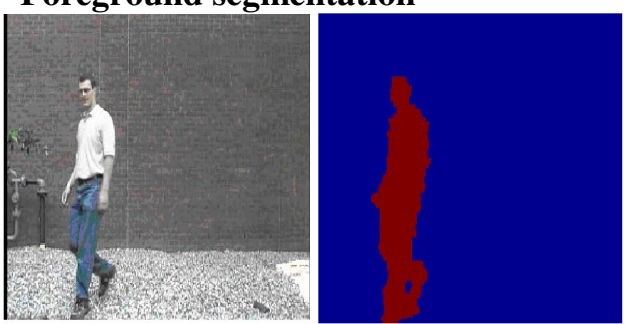

Input Image

Segmentation Maps 
Fuzzy C-means Clustering
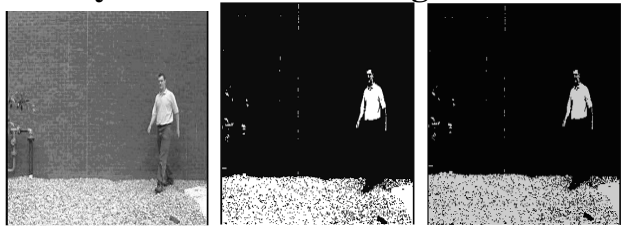

Input Image Segmentation Maps

The final cluster centers are $\operatorname{ccc} 1=103.0929$ and $\operatorname{ccc} 2=213.1369$
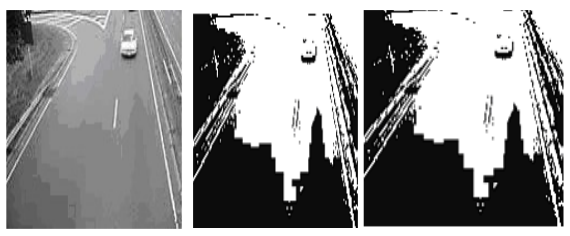

The final cluster centers are $\operatorname{ccc} 1=109.5832$ and $\operatorname{ccc} 2=158.7642$

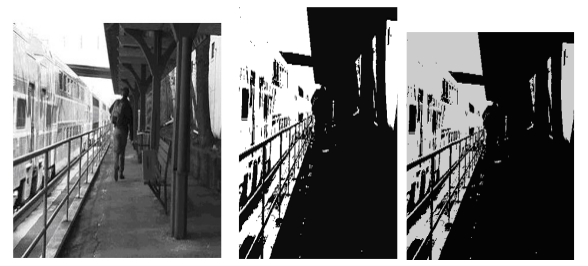

The final cluster centers are $\operatorname{ccc} 1=64.0945$ and $\operatorname{ccc} 2=214.7706$

For Feature extraction the results are classified according to texture, shape, color.

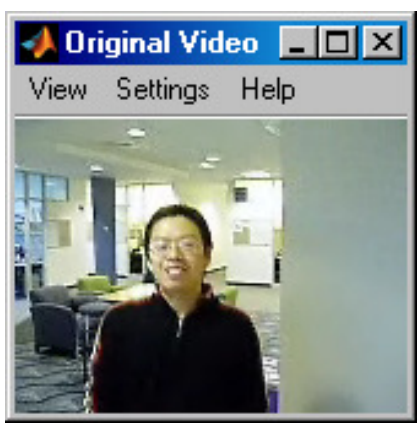

Original Image
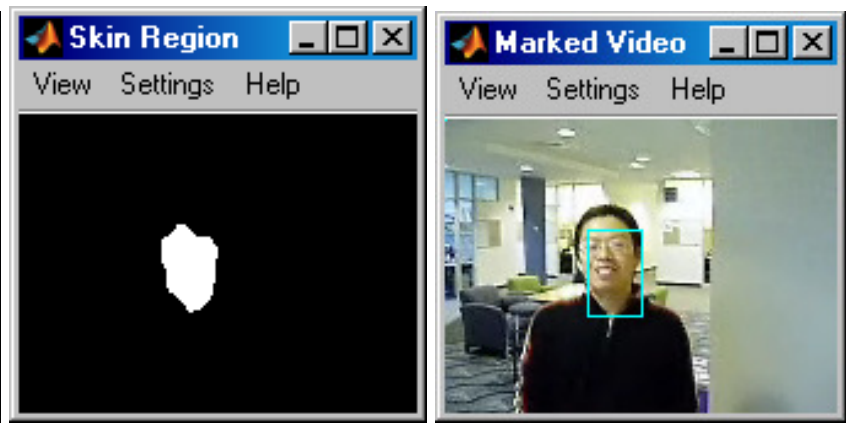

Texture based Edge Detection color based Edge Detection

By comparing the video processing with various algorithms, we conclude that Canny Algorithm is the best for video processing. 

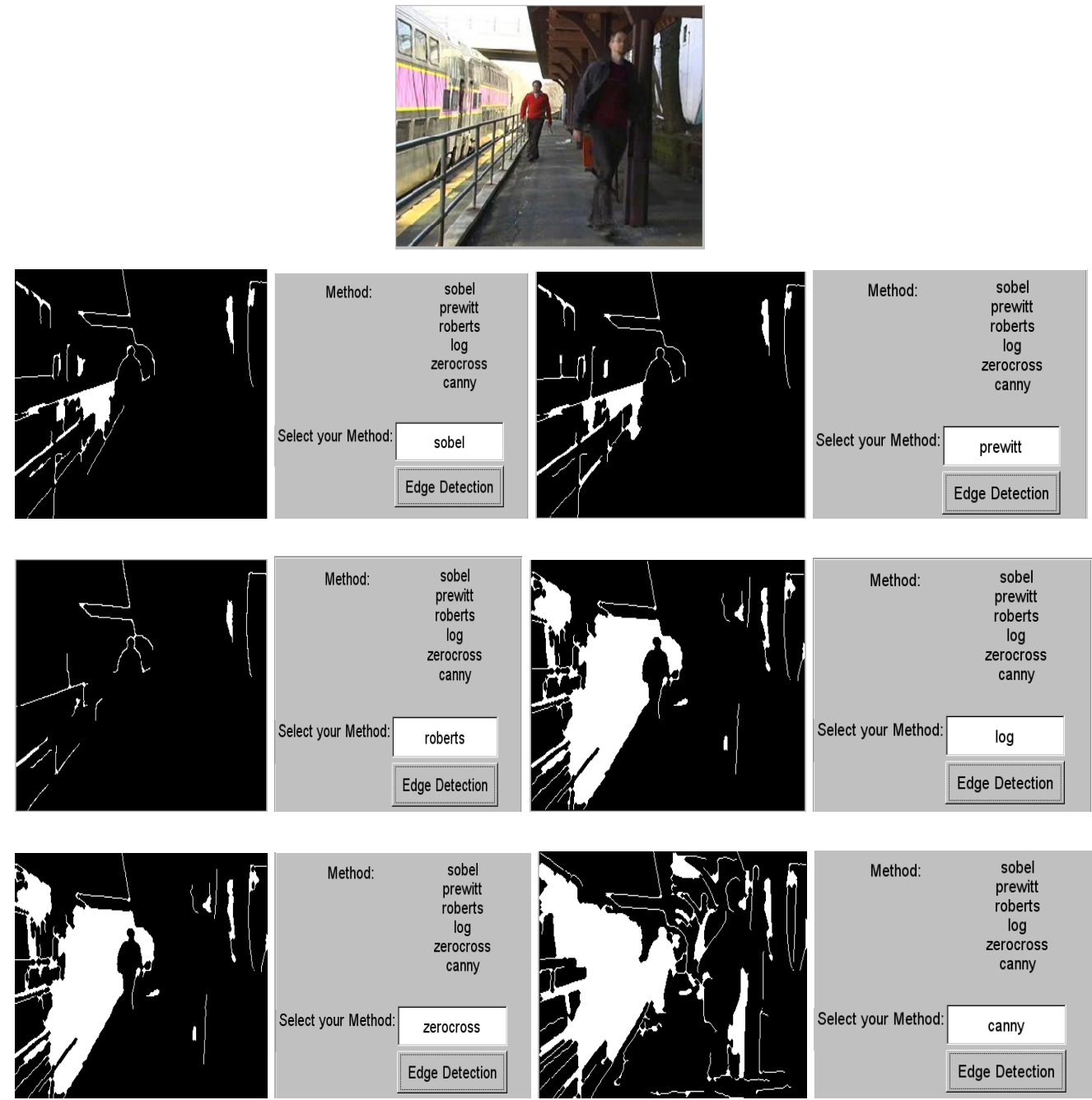

\section{EXPERIMENTAL RESULTS}

The Experimentation data consists of a different videos collected from various sources. The video is preprocessed by denoising technique then the segmentation has been applied on the video frames of individual object detection.

\begin{tabular}{|c|c|c|l|l|l|l|l|c|}
\hline Noise & Filters & AD & SC & MD & NAE & MSE & PSNR & MN CC \\
\hline $\begin{array}{c}\text { Gaussian } \\
\text { Noise }\end{array}$ & $\begin{array}{c}\text { Wiener } \\
\text { Filter }\end{array}$ & 0.8615 & 1.03 & 85.93 & 0.067 & 175.303 & 25.9706 & 0.9798 \\
\hline $\begin{array}{c}\text { Salt \& } \\
\text { Pepper } \\
\text { Noise }\end{array}$ & $\begin{array}{c}\text { Median } \\
\text { Filter }\end{array}$ & 0.4679 & 0.993 & 189.8 & 0.027 & 255.967 & 24.0606 & 0.996 \\
\cline { 2 - 8 } & $\begin{array}{c}\text { Rank } \\
\text { Order }\end{array}$ & 0.3037 & 1.01 & 215.5 & 0.037 & 187.361 & 25.6188 & 0.989 \\
\hline $\begin{array}{c}\text { Periodic } \\
\text { Noise }\end{array}$ & 2D Filter & 0.5665 & 1.024 & 125.9 & 0.1 & 150.026 & 20.2936 & 0.965 \\
\hline
\end{tabular}

Table1: Performance analysis of denoising technique 
Noise reduction technique is the first process in video in order to improve its quality for better results. The denoising technique is applied by introducing various noises such as Gaussian noise, salt and pepper noise and periodic noise on different videos. The test was conducted using various filters and the result shows Wiener filter is best suited for Gaussian noise , Median filter and rank order filter both suits well for salt and pepper noise, for the periodic noise the filter best suits is 2D filter. The performance of these filters are shown in the Table: 1

\section{Performance analysis of segmentation process}

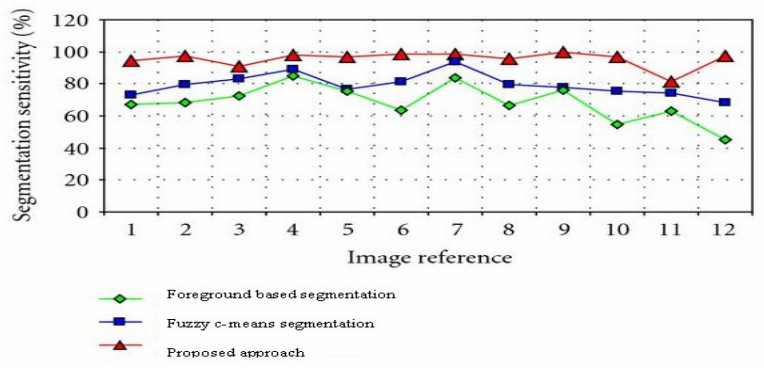

The segmentation approach is ormed on individual frames using three different techniques color segmentation, foreground segmentation and fuzzy c means segmentation on color. The below figure shows the image reference and the segmentation sensitivity of video frames. The result shows fuzzy c means based on color performance better than other two techniques.

Based on various feature extraction on shapes, color, texture we can obtain the image performance can be measured.
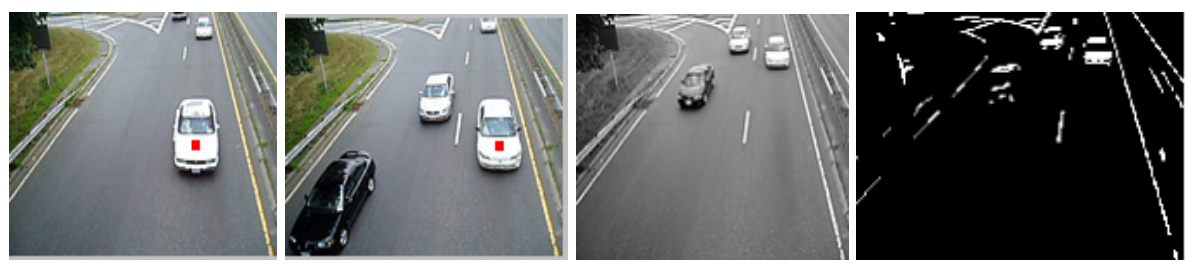

Color Image Edge detected on Shapes Original Image Segmented Image

\section{CONCLUSION}

This paper put forth two important factors of video processing Noise reduction and segmentation. This proposed work reveals which filtering technique is suitable for which type of noise that occur in video. And proposed a new technique of segmentation using Fuzzy-C Means based on color for best segmentation of objects in the color video. To the proposed work further feature extraction takes place at several iteration is measured.Futher it is compared with various algorithms for feature extraction and according to the algorithms compared the result obtained is the canny algorithm is best. To further objects are tracked for further work at which location for different types of objects are identified. 


\section{REFERENCES}

[1] http://www.wisegeek.com/what-is-video-processing.htm.

[2] Leslie Stroebel and Richard D. Zakia (1995). The Focal encyclopedia of photography. Focal Press. p. 507.

[3] Jun Ohta (2008). Smart CMOS Image Sensors and Applications. CRC Press.

[4] Lindsay MacDonald (2006). Digital Heritage. Butterworth- Heinemann.

[5] Junichi Nakamura (2005). Image Sensors and Signal Processing for Digital Still Cameras. CRC Press.

[6] Rafael C. Gonzalez, Richard E. Woods (2007). Digital Image Processing. Pearson Prenctice Hall.

[7] Linda G. Shapiro and George C. Stockman (2001). Computer Vision. Prentice- Hall.

[8] Charles Boncelet (2005). "Image Noise Models". In Alan C. Bovik. Handbook of Image and Video Processing. Academic Press.ISBN 0121197921.

[9] James C. Church, Yixin Chen, and Stephen V. Rice Department of Computer and Information Science, University of Mississippi, "A Spatial Median Filter for Noise Removal in Digital Images", IEEE, page(s): 618- 623, 2008.

[10] Wavelet domain image de-noising by thresholding and Wiener filtering.Kazubek, M. Signal Processing Letters, IEEE, Volume: 10, Issue: 11, Nov. 2003265 Vol.3.

[11] C. Mythili,Dr. V. Kavitha, ."Efficient Technique for Color Image Noise Reduction", T h e R e s e a r c h B u 11 e ti n of J ord a n A C M, V ol. I I (I I I ) Pp | 4 1-44.

[12] Disha Sharma and Gagandeep Jindal," Computer Aided Diagnosis System for Detection of Lung Cancer in CT Scan Images", International Journal of Computer and Electrical Engineering, Vol. 3, No. 5, October 2011-pp: 714-718.

[13] Dana Spiegel," Gesture Recognition System”,5/14/97.

[14] Anil.K.Jain,’Fundamentals of Image processing”, Pearson Prenctice Hall.

[15] M.K. Hu, "Visual pattern recognition by moment invariants," IRE Trans.on Information Theory, 8, pp. 179-187, 1962. 